\title{
Tunable narrow linewidth AIGaInP semiconductor disk laser for Sr atom cooling applications
}

\author{
David Pabeuf And Jennifer E. Hastie
}

Institute of Photonics, Department of Physics, University of Strathclyde, The Technology and Innovation Centre, 99 George Street, Glasgow

*Corresponding author: david.paboeuf@strath.ac.uk

Received XX Month XXXX; revised XX Month, XXXX; accepted XX Month XXXX; posted XX Month XXXX (Doc. ID XXXXX); published XX Month XXXX

We report a frequency stabilised semiconductor disk lasers based on AlGaInP and operating at $689 \mathrm{~nm}$, a wavelength of interest for atomic clocks based on strontium atoms. With a gain structure designed for emission at around $690 \mathrm{~nm}$, more than $100 \mathrm{~mW}$ of output power was generated in single frequency operation. We show that the source can be tuned over $8 \mathrm{~nm}$ with picometer precision. By servo-locking the frequency to the side of fringe of a reference cavity, we demonstrate rms frequency noise of $5.2 \mathrm{kHz}$.

OCIS codes: (140.7270) Vertical emitting lasers; (140.3425) Laser stabilization; (140.7300) Visible lasers

http://dx.doi.org/10.1364/AO.99.099999

\section{INTRODUCTION}

Semiconductor disk lasers (SDLs) have great potential for optical clock applications. They benefit from a high finesse external cavity, hence a long photon lifetime, and have a low carrier lifetime (few ns). Thus, the laser dynamics are unique among semiconductor lasers, allowing for low intensity noise (so-called class A behaviour) and a sub-Hz Schawlow-Townes limit [1]. This is in contrast to conventional diode lasers in external cavities which photon lifetime is much shorter and typical Schawlow-Townes limit is $\sim 100 \mathrm{kHz}$ [2]. Also, as for any solid state lasers the frequency noise bandwidth of SDLs is in the $\mathrm{kHz}$ range, hence about 3 orders of magnitude smaller than the one of dye lasers or laser diode. These outstanding properties, and the fact that they can be designed to operate at almost any wavelength from the visible to the midinfrared via bandgap engineering and efficient intracavity conversion [3,4], make SDLs ideal laser sources for optical clocks and atom trapping [5]. Indeed, there are already a few instances of SDL development for atomic clock applications targeting clocks based on either caesium [6,7] or mercury [8,9].

Among optical clocks, those based on strontium are prime candidates for new optical standards for the definition of the second [10]. Indeed, recently an Sr atomic clock demonstrated record accuracy and stability at the $10^{-18}$ level [11]. Operating such clocks requires several laser beams with wavelengths lying within the $670-700 \mathrm{~nm}$ spectral range, which is accessible with lasers based on GaInP quantum wells. In particular, $679 \mathrm{~nm}, 689 \mathrm{~nm}$ and $698 \mathrm{~nm}$ are respectively a re-pumping transition, the second stage cooling transition and the clock transition for neutral $\mathrm{Sr}[12,13]$. For these applications the power requirements are usually a few 100s of milliwatts; the most strenuous requirements are for the linewidths, which should be in the $\mathrm{kHz}$ range for the cooling and re-pumping lasers and as narrow as possible (sub Hz) for the clock laser.
In this paper we report a step towards SDL development for Sr optical clocks with the demonstration of an SDL designed for emission at $689 \mathrm{~nm}$, the wavelength required for the second stage cooling transition in $\mathrm{Sr}$, and frequency stabilised in the $\mathrm{kHz}$ range relative to a reference Fabry-Perot cavity. We also show a simple way to tune the wavelength to any arbitrary value within the tuning range of the source, which is of tremendous importance in the context of atom cooling since specific absorption lines are targeted.

\section{DESCRIPTION OF THE LASER CAVITY}

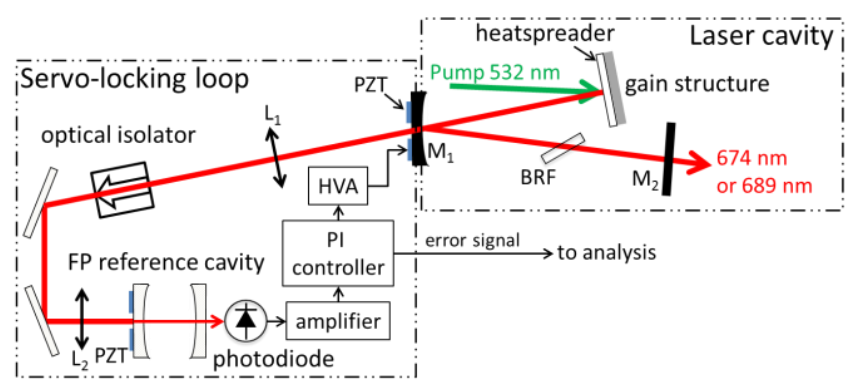

Fig. 1 Schematic of the single-frequency laser cavity and of the servolocking loop. M1,M2, cavity mirrors; BRF, birefringent filter; PZT, piezoelectric transducer; FP, Fabry-Perot; PI, proportional integrator; HVA, high voltage amplifier.

The design of the SDL gain structure is similar to those we have previously reported [14]. It contains ten pairs of strained GaInP quantum wells, embedded in AlGaInP barriers and evenly distributed at the antinode positions of the resonant internal optical field. The active region is grown on top of an AlGaAs distributed Bragg reflector (DBR). The structure is optimised (quantum well peak gain, resonant 
periodic gain wavelength and DBR high reflectivity stop band) for laser operation at $\sim 689 \mathrm{~nm}$ and is pumped with up to $3 \mathrm{~W}$ at $532 \mathrm{~nm}$ in a $90 \mu \mathrm{m}$ diameter focal spot. Since noise transfer from the pump can have a detrimental effect on the linewidth of the laser, we use a low intensity noise pump laser (relative intensity noise specified as < $0.02 \%$ by the manufacturer).

The laser cavity, shown in Fig. 1, consists of 3 mirrors: the DBR of the gain structure, a highly reflective (R>99.9\%) folding mirror (M1) with a radius of curvature equal to $100 \mathrm{~mm}$ and a plane output coupler (M2). The output coupling value is $1.8 \%$ at $689 \mathrm{~nm}$. The total length of the cavity is about $200 \mathrm{~mm}$ and the distance between the folding mirror and the semiconductor disk is $53 \mathrm{~mm}$. The cavity mode at the gain medium is slightly elliptical with diameters $2 \mathrm{w}=80$ and $90 \mu \mathrm{m}$. A $500-\mu \mathrm{m}$-thick uncoated single-crystal diamond heat-spreader is bonded to the semiconductor disk for thermal management. A 6-mmthick quartz plate acting as a birefringent filter (BRF) is inserted at Brewster's angle inside the cavity. This allows tuning of the wavelength over a relatively broad range: $685-693 \mathrm{~nm}$. It should be noted that the tuning range is limited by the free spectral range of the BRF and not by the gain structure. Due to the Fabry-Perot effect inside the heatspreader, the tuning is done in steps of about $180 \mathrm{pm}$, which corresponds to the free spectral range of a $500-\mu \mathrm{m}$-thick diamond etalon. This effect and ways to circumvent the tuning limitation it imposes are discussed in section 5 .

Single frequency emission is achieved via the combination of all the intracavity elements and careful optimisation of the cavity length. Indeed, the Fabry-Perot effect due to the heatspreader is beneficial and enhances the mode filtering. Once the cavity is optimized, repeatable single frequency operation is routinely obtained as evidenced in Fig. 2.

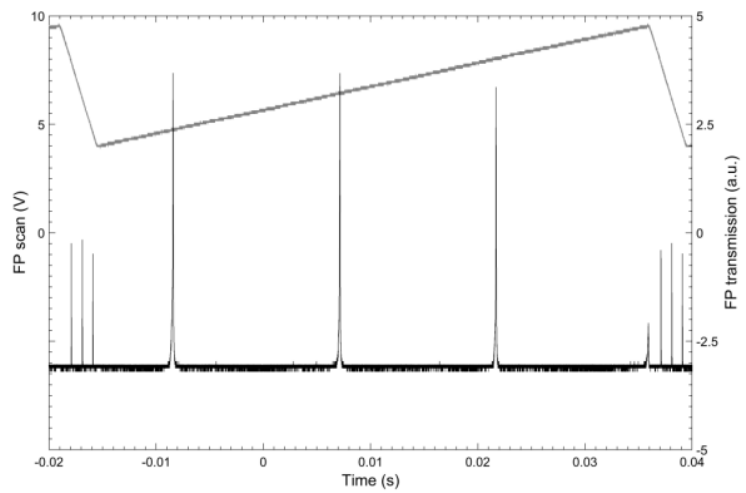

Fig. 2. Characterisation of the single frequency emission with a scanned Fabry-Perot (FP) cavity. The free spectral range of the confocal cavity is $300 \mathrm{MHz}$. The measurement is for an incident pump power of $3 \mathrm{~W}$ and a laser power of $80 \mathrm{~mW}$ at $689 \mathrm{~nm}$.

\section{DESCRIPTION OF THE LOCKING SETUP}

A schematic of our locking setup, a classic 'side of fringe' scheme [15], is shown on the left side of Fig. 1. The frequency reference is a confocal Fabry-Perot (FP) cavity with a free spectral range of $300 \mathrm{MHz}$ (Melles Griot 13SAE005), made of Invar for a reduced sensitivity to thermal fluctuations. We use one of the low power beams leaking through folding mirror M1 for the frequency locking. The beam is first collimated and then focused inside the reference cavity. An optical isolator with $30 \mathrm{~dB}$ isolation ratio is placed between the laser and the FP cavity to avoid any perturbing feedback to the laser cavity. After optimisation of the mode matching, the finesse of the Fabry-Perot cavity is measured as 290 (see Fig. 2), which results in a resonance peak width slightly larger than $1 \mathrm{MHz}$. Full details of the experimental conditions, including the resulting frequency noise detection sensitivity used in the locking experiments shown in the rest of this paper, are given in Table 1 . The detection bandwidth of the photodiode and the amplifier is $30 \mathrm{kHz}$, which is an order of magnitude larger than the typical noise bandwidth for such a laser [16].

The error signal of the servo-loop is generated by comparing the signal transmitted by the FP cavity and a command set to half of the maximum of its resonance peak. The correction signal is then delivered by a proportional integral controller (Newport LB 1005) and amplified by a large bandwidth high voltage amplifier (Piezodrive PDX 150b). The frequency fluctuations are then compensated via a piezoelectric transducer (PI P-010.00H) upon which the folding mirror (M1 in Fig 1) is fixed with paraffin wax.

In order to achieve a large correction bandwidth, we used a mirror with reduced size and weight: 2-mm-thick, 10-mm-diameter. The resulting resonance frequency of the ensemble is $\sim 100 \mathrm{kHz}$, which is about 3 times larger than that measured with a standard 6-mm thick, half-inch mirror. In order to avoid exciting that resonance, the correction bandwidth of the servo loop is limited to $5 \mathrm{kHz}$ with an extra low pass filter. Such a bandwidth roughly corresponds to the frequency noise bandwidth of the free running laser. Free running frequency noise measurement of a similar laser has already been reported in [17]. It should be noted that such a low frequency noise bandwidth is a typical feature of solid-state lasers in general and SDLs in particular [16].

\begin{tabular}{cccc}
\hline Wavelength & Finesse & $\begin{array}{c}\text { Transmission } \\
\text { signal }\end{array}$ & $\begin{array}{c}\text { Frequency } \\
\text { discriminator }\end{array}$ \\
\hline $689 \mathrm{~nm}$ & 290 & $6.9 \mathrm{~V}$ & $6.7 \mathrm{~V}_{\mathrm{MHz}}^{-1}$ \\
\hline
\end{tabular}

Table 1. Experimental conditions under which frequency locking was achieved. The free spectral range of the reference cavity is 300 MHz.

\section{FREQUENCY NOISE CHARACTERISATION}

Once the locking loop is closed, the laser frequency remains locked to the FP cavity for several minutes: the limit being the drift of the reference cavity, which at some point induces a mode hop of the laser. From the residual error signal of the servo loop, one can deduce the relative root mean square (rms) frequency noise of the locked laser. The resulting frequency noise power spectral density (PSD) is shown in Fig 3. We obtained a relative rms frequency noise of $5.2 \mathrm{kHz}$. The PSD is mostly flat for the whole correction bandwidth (up to $5 \mathrm{kHz}$ ) apart from some localised peaks attributed to residual mechanical resonances of the laser cavity. The broader peaks located at frequencies higher than $10 \mathrm{kHz}$ are due to electronic oscillations, which we kept as low as possible by limiting the gain of the loop. These oscillations are one of the current limitations of our setup as they prevent an increase of the gain of the loop and thus further reduction of the frequency noise in the low frequency band. Calculating the square root of the integral of the PSD within a given frequency bandwidth allows an estimate to be made of the contribution of that specific bandwidth to the overall noise. From this we can tell that frequencies below $10 \mathrm{kHz}$ contributes about $80 \%$ of the frequency noise and that in particular the $0.1-1 \mathrm{kHz}$ decade is responsible for more than $40 \%$. Noise within this band is usually due to mechanical instabilities. We believe that with an improved insulation and a monolithic setup, we should be able to reduce the mechanical noise and achieve a lower frequency noise level. 


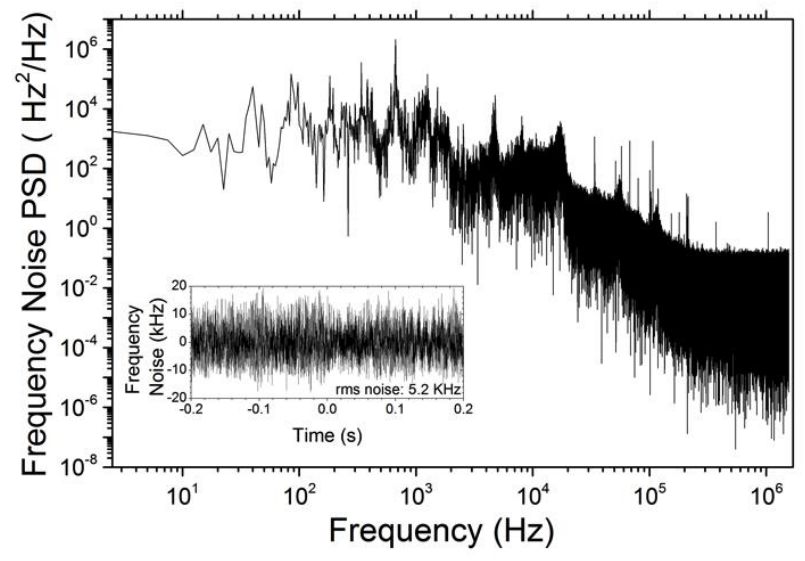

Fig. 3. Frequency noise power spectral density calculated from the residual error signal of the servo locking loop (shown in the inset) measured over $400 \mathrm{~ms}$ with the laser operating at $689 \mathrm{~nm}, \mathrm{P}=80 \mathrm{~mW}$ $\left(\mathrm{P}_{\text {pump }}=3 \mathrm{~W}\right)$.

It should be noted that the relative linewidth calculated from the rms frequency noise, while the commonly reported performance parameter for such SDL systems, is not equivalent to the laser linewidth. From the knowledge of the frequency noise PSD one can, however, deduce the shape of the optical field and the actual relative linewidth of the laser. Indeed one can show via the Wiener-Khintchine theorem, assuming that the phase noise follows a normal distribution, that the autocorrelation function of the field $R(\tau)$ can be expressed as [18-20]:

$$
R(\tau)=E_{0}{ }^{2} e^{-i 2 \pi v_{0} \tau} \times \exp \left(-\int_{0}^{\infty} S_{v}(f) \frac{1-\cos 2 \pi f \tau}{f^{2}} d f\right)
$$

where $\mathrm{E}_{0}$ is the field amplitude and $v_{0}$ the optical frequency. Here $\mathrm{S}_{v}(\mathrm{f})$, the frequency noise PSD, is considered as single-sided to exactly correspond to the values plotted in Fig. 3. Once the field autocorrelation is determined, the laser line shape can be obtained by applying the Wiener-Khintchine theorem again and computing the fast Fourier transform of $\mathrm{R}(\tau)$.

By applying Eq. 1 to the frequency noise PSD of Fig. 3, the field autocorrelation shown in Fig. 4 a) can be obtained. Computing the Fourier transform of the autocorrelation leads to the laser linewidth shown in Fig. 4. In order to limit the computation requirements we limited the bandwidth of the PSD to $100 \mathrm{kHz}$. The noise level above this frequency is, in any case, very low and limited by our noise detection floor. Moreover, frequency noise at high frequencies only contributes to the wings of the laser line and not to its width [20]. The sampling parameters used for the calculations are given in the figure caption. From this, we deduce a laser linewidth of $11.4 \mathrm{kHz}$. The contribution of the oscillation peak of the PSD around $10 \mathrm{kHz}$ can be seen in the appearance of a small pedestal on the optical field. This doesn't contribute significantly to the linewidth since it is a relatively high frequency noise component.
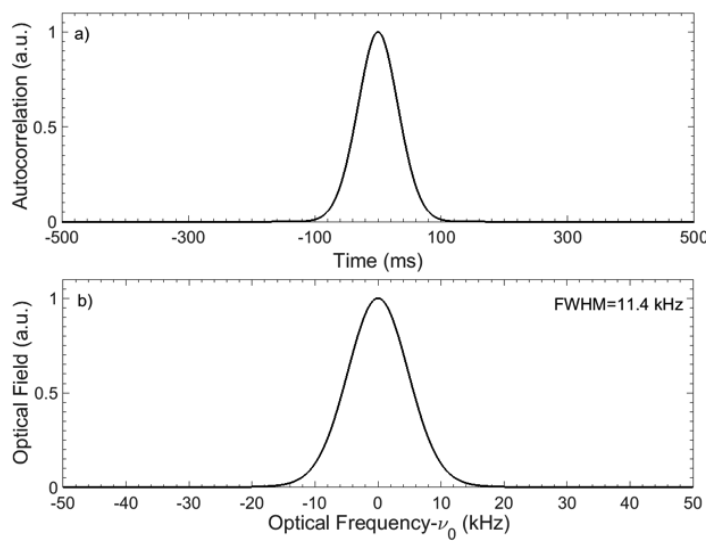

Fig. 4. a) Autocorrelation of the optical field calculated from the frequency noise PSD. b) Power spectral density of the optical field. The time sample used for the calculation of the autocorrelation was $3 \mathrm{~ms}$ and the frequency sample for the optical field was $3 \mathrm{~Hz}$.

\section{FINE WAVELENGTH TUNING}

As mentioned earlier, the Fabry Perot effect caused by the diamond heatspreader only allows the laser to oscillate at wavelengths matching its free spectral range. This is exemplified in Fig. 5 a) which shows that the SDL is indeed tuned by steps equal to the free spectral range of the heatspreader $(\sim 180 \mathrm{pm})$. This can be a crucial issue in the context of atom cooling as one need to use very specific wavelengths, which, for a given heatspreader, may not be available.

If one wants to tune an SDL to any arbitrary wavelength, several solutions can be implemented. The first one consists of placing the heatspreader on the back of the SDL sample, hence outside of the cavity. This requires chemical etching processes to thin down the substrate in order to reduce the thermal resistance of the gain chip. This approach, is very efficient for InGaAs based SDLs [21] where the gain structures have a high overall thermal conductivity. In our case, the thermal conductivity of AlGaInP is much lower and the most efficient way to extract the heat is by use of an intracavity heatspreader [22,23]. Another solution consists of using a wedged heatspreader. If the wedge angle is large enough, typically 2 to 3 degrees [22], the etalon effect can be canceled out and one can continuously tune the wavelength. This solution, though, requires the deposition of antireflection coatings on the surface of the heatspreader. All these solutions basically consist in getting rid of the Fabry-Perot effect of the heatspreader. Since in our case the single frequency emission relies on the filtering from the heatspreader, we chose to keep it as it is inside the cavity. As an alternative way of tuning the wavelength, we use its residual wedge which is large enough (specified as $<1 \mathrm{mrad}$ by the manufacturer) to make it behave like a spatially tunable etalon. As a result, by translating the \{sample + heatspreader ensemble one can tune the wavelength. This is shown in Fig. 5 b), for which the wavelength of the laser was recorded with a wavemeter while the sample mount was manually translated via a translation stage. One can clearly see that the wavelength linearly follows the position of the ensemble. Moving it by about $550 \mu \mathrm{m}$ enables coverage of an entire free spectral range of the heatspreader. Fig. 5 c) shows that translating the ensemble by about $10 \mu \mathrm{m}$ permits picometer precision on the output wavelength as the laser jump from one longitudinal mode of the laser cavity to the next. 

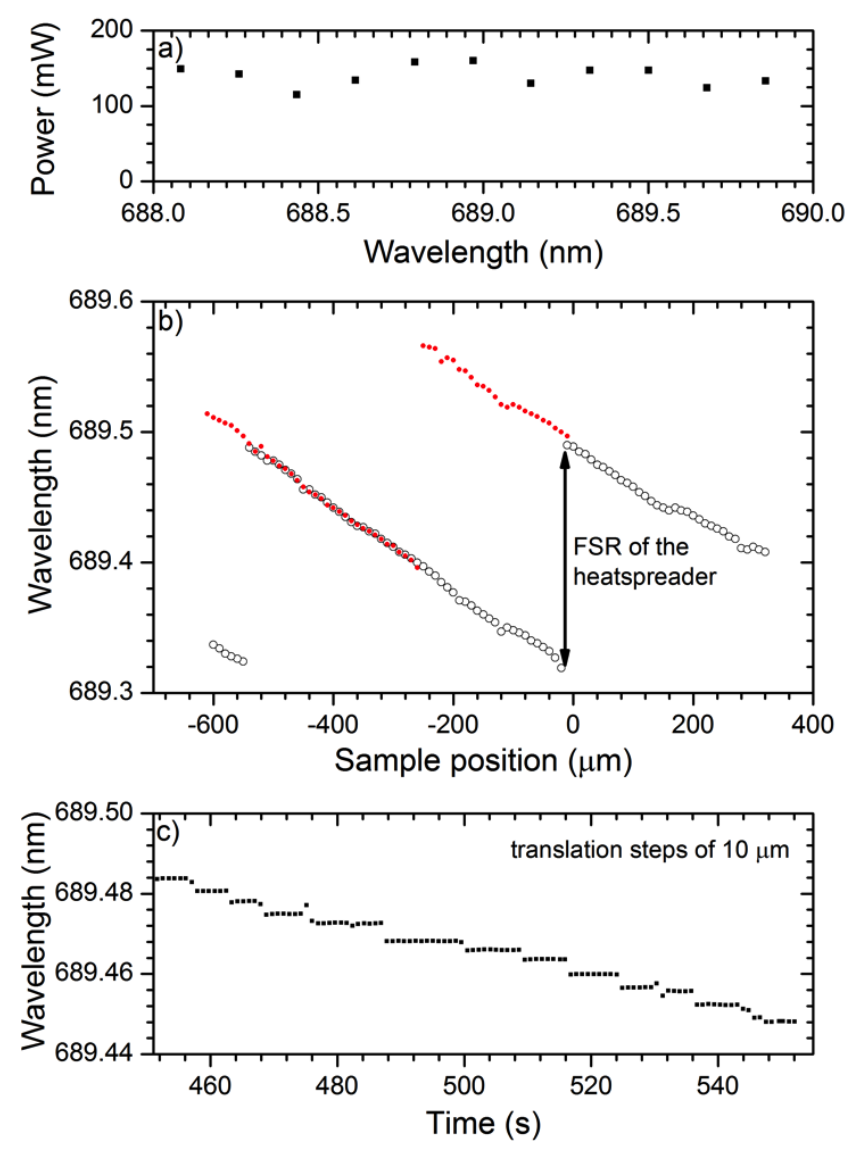

Fig. 5. Tuning characteristics. a) Broad tuning achieved by rotation of the BRF b) Coarse tuning achieved by translation of the ensemble gain chip + diamond heatspreader. The figure shows two consecutive measurements made for two different orientations of the BRF. c) Fine tuning. The wavelength was recorded vs time while the sample was translated by steps of about $10 \mu \mathrm{m}$. As a side note, measuring the slope of figure $5 \mathrm{~b}$ ) leads to an estimated wedge of $0.23 \mathrm{mrad}$ for the diamond heatspreader.

During the tuning process, optimization of the output power can be done by finely rotating the BRF to shift its maximum transmission to the operating wavelength. The orientation of the BRF is actually crucial since it also defines the position for which the wavelength will switch from one resonance of the heatspreader to the next. This is evidenced on Figure $5 \mathrm{~b}$ ), which shows two consecutive tuning measurements but with a different orientation for the BRF.

As a result one can make the laser operate at any arbitrary wavelength between 685 and $693 \mathrm{~nm}$ by first rotating the BRF to reach the closest etalon peak and then carefully translating the gain chip to move that peak to the desired wavelength. By finely readjusting the BRF one can optimize the power and reach the $100 \mathrm{~mW}$ level.

\section{CONCLUSION}

We have demonstrated a tunable, single frequency semiconductor disk laser with AlGaInP gain structures designed to address those wavelengths in the red spectral region required for Sr based optical clocks. Output powers above $100 \mathrm{~mW}$ were obtained at $689 \mathrm{~nm}$. The $\mathrm{rms}$ frequency noise was reduced to a few $\mathrm{kHz}$ over a $400 \mathrm{~ms}$ sampling time by locking the frequency to a reference Fabry-Perot cavity via the side-of-fringe technique. Calculations of the relative laser linewidths lead to values just over $10 \mathrm{kHz}$. We also show that the wavelength of the laser can be arbitrarily set to any value within a 685 to $693 \mathrm{~nm}$ range.

These results are very close to the requirements for cooling $\mathrm{Sr}$ atoms, for which the transition linewidth at $689 \mathrm{~nm}$ is $7 \mathrm{kHz}$. More experiments and characterization aiming at decreasing the linewidth and using the laser in an atom cooling setup are currently under way. One way to improve the linewidth would be to combine a more stable, vibration isolated, monolithic setup with a high-finesse temperature controlled reference cavity maintained in vacuum. The latter would eliminate the frequency drift and allow for much longer stabilization sequences. One should also note that these results demonstrate the great capabilities of SDL in general and of the interest of AlGaInP based ones in particular. For Sr and Sr+ clocks, many of the wavelengths of interest are within the accessible range of GaInP. By adjusting the gain medium compositions one should be able to provide a full range of SDL based sources to the Sr atomic clock community.

Funding Information. This work was funded by the UK Engineering and Physical Sciences Research Council (EPSRC) under grant EP/I022791/1 and the National Quantum Technology Hub for Sensors and Metrology (EP/M013294/1).

Acknowledgment. The laser gain structures were grown by Andrey Krysa at the EPSRC National Centre for III-V Technologies.

\section{Dataset.}

Data related to this publication have been made available at the University of Strathclyde data repository. http://dx.doi.org/10.15129/2eb89c49-04fc-485e-a22e$1 \mathrm{acb} 896 \mathrm{e} 150 \mathrm{~d}$.

\section{References}

1. A. Garnache, A. Ouvrard, and D. Romanini, "Single-Frequency operation of External-Cavity VCSELs: Non-linear multimode temporal dynamics and quantum limit.," Opt. Express 15, 9403 (2007).

2. A. Garnache, A. Laurain, M. Myara, J.-P. Perez, L. Cerutti, A. Michon, G. Beaudoin, I. Sagnes, P. Cermak, and D. Romanini, "Design and properties of high-power highly coherent singlefrequency VECSEL emitting in the near- to mid-IR for photonic applications," in Proceedings of SPIE, U. Keller, ed., Photonics West (SPIE - Society of Photolnstrumentations Engineers, 2011), Vol. 7919, pp. 791914-791914-11.

3. N. Schulz, J. M. Hopkins, M. Rattunde, D. Burns, and J. Wagner, "High-brightness long-wavelength semiconductor disk lasers," Laser Photonics Rev. 2, 160-181 (2008).

4. S. Calvez, J. E. Hastie, M. Guina, O. G. Okhotnikov, and M. D. Dawson, "Semiconductor disk lasers for the generation of visible and ultraviolet radiation," Laser Photon. Rev. 3, 407-434 (2009).

5. S. Burd, D. Leibfried, a. C. Wilson, and D. J. Wineland, "Optically pumped semiconductor lasers for atomic and molecular physics," in Proceedings of SPIE, M. Guina, ed. (2015), Vol. 9349, p. 93490P.

6. B. Cocquelin, D. Holleville, G. Lucas-Leclin, I. Sagnes, A. Garnache, M. Myara, and P. Georges, "Tunable single-frequency operation of a diode-pumped vertical external-cavity laser at the cesium D2 line," Appl. Phys. B 95, 315-321 (2009).

7. P. Dumont, F. A. Camargo, J.-M. Danet, D. Holleville, S. Guerandel, G. Pillet, G. Baili, L. Morvan, D. Dolfi, I. Gozhyk, G. Beaudoin, I. Sagnes, P. Georges, and G. Lucas-Leclin, "Low-Noise Dual-Frequency Laser for Compact Cs Atomic Clocks," J. Light. Technol. 32, 3817-3823 (2014).

8. J. R. Paul, Y. Kaneda, T. Wang, C. R. Lytle, J. V. Moloney, and R. J. 
Jones, "Doppler-free spectroscopy of mercury at $253.7 \mathrm{~nm}$ using a high-power, frequency-quadrupled, optically pumped externalcavity semiconductor laser," Opt. Lett. 36, 61-63 (2010).

9. J. R. Paul, C. R. Lytle, Y. Kaneda, J. V. Moloney, T.-L. Wang, and R. J. Jones, "Optically-pumped external-cavity semiconductor lasers for precision spectroscopy and laser cooling of atomic $\mathrm{Hg}$," in Proceedings of SPIE, J. E. Hastie, ed. (2013), p. 86060R-86060R9.

10. P. Gill, "When should we change the definition of the second?," Philos. Trans. A. Math. Phys. Eng. Sci. 369, 4109-30 (2011).

11. M. M. Boyd, T. L. Nicholson, J. R. Williams, S. L. Campbell, M. Bishof, X. Zhang, W. Zhang, S. L. Bromley, and J. Ye, "An optical lattice clock with accuracy and stability at the 10(-18) level.," Nature 506, 71-5 (2014).

12. X. Xu, T. H. Loftus, J. L. Hall, A. Gallagher, and J. Ye, "Cooling and trapping of atomic strontium," J. Opt. Soc. Am. B 20, 968 (2003).

13. I. Courtillot, A. Quessada-Vial, A. Brusch, D. Kolker, G. D. Rovera, and P. Lemonde, "Accurate spectroscopy of Sr atoms," Eur. Phys. J. D 33, 161-171 (2005).

14. J. E. Hastie, S. Calvez, M. D. Dawson, T. Leinonen, A. Laakso, J. Lyytikäinen, and M. Pessa, "High power CW red VECSEL with linearly polarized TEMOO output beam," Opt. Express 13, 77 (2005).

15. R. L. Barger, M. S. Sorem, and J. L. Hall, "Frequency stabilization of a cw dye laser," Appl. Phys. Lett. 22, 573-575 (1973).

16. A. Laurain, C. Mart, J. Hader, J. V. Moloney, B. Kunert, and W. Stolz, "Optical noise of stabilized high-power single frequency optically pumped semiconductor laser," Opt. Lett. 39, 1573 (2014).

17. D. Pabœuf, P. J. Schlosser, and J. E. Hastie, "Frequency stabilization of an ultraviolet semiconductor disk laser," Opt. Lett. 38, 1736 (2013).

18. D. Elliott, R. Roy, and S. Smith, "Extracavity laser band-shape and bandwidth modification," Phys. Rev. A 26, 12-18 (1982).

19. C. Chardonnet, Laser Monofréquence et Stabilisation (EDP Sciences, 1996).

20. G. Di Domenico, S. Schilt, and P. Thomann, "Simple approach to the relation between laser frequency noise and laser line shape," Appl. Opt. 49, 4801 (2010).

21. S. Lutgen, T. Albrecht, P. Brick, W. Reill, J. Luft, and W. Späth, "8W high-efficiency continuous-wave semiconductor disk laser at 1000 nm," Appl. Phys. Lett. 82, 3620 (2003).

22. A. J. Maclean, A. J. Kemp, S. Calvez, J.-Y. Kim, T. Kim, M. D. Dawson, and D. Burns, "Continuous Tuning and Efficient Intracavity Second-Harmonic Generation in a Semiconductor Disk Laser With an Intracavity Diamond Heatspreader," IEEE J. Quantum Electron. 44, 216-225 (2008).

23. A. J. Maclean, R. B. Birch, P. W. Roth, A. J. Kemp, and D. Burns, "Limits on efficiency and power scaling in semiconductor disk lasers with diamond heatspreaders," J. Opt. Soc. Am. B 26, 2228 (2009). 